KCL-TH-97-55

hep-th/9710034

September 1997

\title{
Classical M-Fivebrane Dynamics and Quantum $N=2$ Yang-Mills
}

\author{
P.S. Howe \\ N.D. Lambert \\ and \\ P.C. West ${ }^{\star}$ \\ Department of Mathematics \\ King's College, London \\ England \\ WC2R 2LS
}

\begin{abstract}
We obtain the complete quantum Seiberg-Witten effective action for $N=2$ supersymmetric $S U(N)$ Yang-Mills theory from the classical M-fivebrane equations of motion with $N$ threebranes moving in its worldvolume.
\end{abstract}

^ phowe, lambert, pwest@mth.kcl.ac.uk 


\section{Introduction}

The remarkable solution of Seiberg and Witten [1] for the complete nonperturbative chiral part of the effective action of $S U(2) N=2$ Yang-Mills theory spontaneously broken to $U(1)$ represents one of the few examples in quantum field theory where the complete quantum effects are known. The result was derived using essentially two inputs; the first was the complete perturbative result for this action which had been known for many years $[2,3]$ and the second was an application of electromagnetic duality [4]. Since only the first of these results is well established there have been a number of attempts to give alternative derivations of the Seiberg-Witten effective action. Some of the terms in the effective action have been confirmed using instanton corrections [5]. It has also been observed that the Seiberg-Witten effective action obeys a particular non-trivial relation [6], which has been shown to be a consequence of the anomalous $N=2$ superconformal Ward identities of a spontaneously broken $N=2$ Yang-Mills theory [7]. It has since been argued [8] that this relation implies the full effective action.

One of the most mysterious features of the Seiberg-Witten effective action is the way that it is related to an associated Riemann surface. In particular it was shown how the effective action could be constructed given the Riemann surface using a specific recipe. However, how this surface arises naturally in the theory was not apparent. Shortly after the appearance of references [1] Riemann surfaces that were thought to correspond to the spontaneously broken gauge groups $S U(N)$ were proposed [9].

An alternative approach has been to attempt to derive the Seiberg-Witten results from a string theory. It was found in [10] that the Seiberg-Witten curves occured in Calabi-Yau Compactifications of type IIB string theory and furthermore, from the $\mathrm{M}$ theory perspective, these could be interpreted as a single fivebrane wrapped on a Riemann surface. More recently, Witten [11] considered configurations of intersecting NS-fivebranes and D-fourbranes in IIA string theory. He argued that the spontaneously broken $N=2$ Yang-Mills theory appeared on the 
parallel D-fourbranes. By embedding this picture in $\mathrm{M}$ theory he argued that the configuration could be represented by a single M-fivebrane and was able to show that the Riemann surfaces which arise in not only the $S U(2)$ theory, but also in its $S U(N)$ generalisations, appear in a very natural way. However, in this work the connection between the M-fivebrane dynamics and the final Seiberg-Witten effective action remained obscure.

In this paper, we shall use the M-theory fivebrane classical dynamics to derive the Seiberg-Witten effective action. In particular we use the M-fivebrane dynamics as formulated in [12], although there are other formulations [13]. It was recently shown that the M-fivebrane admits onebrane [14] and threebrane solutions [15] within it. We will consider a single M-fivebrane on which some threebranes are moving. The zero modes of this configuration correspond to the modes of the spontaneously broken $N=2$ Yang-Mills theory which can be viewed as living on the four-dimensional worldvolume of a threebrane. It is a consequence of the Bogomoln'yi condition for the threebranes that the M-fivebrane can be viewed as being wrapped on a Riemann surface which is itself embedded in a four-dimensional space. This latter space is composed of the two dimensions of the M-fivebrane transverse to the threebranes and the active two of the five transverse dimensions of the fivebrane embedded in $\mathrm{M}$ theory. We consider the classical fivebrane action for threebrane configurations in which the zero modes are allowed to depend on the threebrane coordinates. We show that it is precisely the same as the full non-perturbative Seiberg-Witten effective action for spontaneously broken $S U(N)$ Yang-Mills gauge theory. In practice we only carry out this calculation for the scalars, but it follows from $N=2$ supersymmetry that it holds for the full action involving the fermions and vectors. 


\section{The Threebrane Effective Action}

The $\mathrm{M}$ theory fivebrane has a six-dimensional $(2,0)$ tensor multiplet of massless fields on its worldvolume. The classical equations of motion in the absence of fermions and background fields are [12]

$$
\begin{aligned}
G^{\hat{m} \hat{n}} \nabla_{\hat{m}} \nabla_{\hat{n}} X^{a^{\prime}} & =0 \\
G^{\hat{m} \hat{n}} \nabla_{\hat{m}} H_{\hat{n} \hat{p} \hat{q}} & =0,
\end{aligned}
$$

where the worldvolume indices are $\hat{m}, \hat{n}=0,1, \ldots, 5$ and the transverse indices are $a^{\prime}, b^{\prime}=6,7,8,9,10$. In (2.1) $\nabla$ is the Levi-Civita connection of the metric $g_{\hat{m} \hat{n}}=\eta_{\hat{m} \hat{n}}+\partial_{\hat{m}} X^{a^{\prime}} \partial_{\hat{n}} X^{a^{\prime}}$. The three form $H_{\hat{m} \hat{n} \hat{p}}$ is closed but not self-dual. Rather, in the vielbein frame defined by $E_{\hat{a}} \hat{m}, \hat{a}, \hat{b}=0,1, \ldots, 5$, it is related to the self-dual three form $h_{\hat{a} \hat{b} \hat{c}}$ via $H_{\hat{a} \hat{b} \hat{c}}=m_{\hat{a}}^{\hat{d}} m_{\hat{b}}^{\hat{e}} h_{\hat{d} \hat{e} \hat{c}}$, where $m_{\hat{a}}^{\hat{b}}=\delta_{\hat{a}}^{\hat{b}}-2 h_{\hat{a} \hat{c} \hat{d}} h^{\hat{b} \hat{c} \hat{d}}$. The vielbein $E_{\hat{a}}^{\hat{m}}$ is associated to the metric $G^{\hat{m} \hat{n}}$ through $G^{\hat{m} \hat{n}}=E_{\hat{a}}{ }^{\hat{m}} E_{\hat{b}}{ }^{\hat{n}} \eta^{\hat{a} \hat{b}}$ but the metric $g^{\hat{m} \hat{n}}$ is obtained from the vielbein $e_{\hat{a}}^{\hat{m}}=\left(m^{-1}\right)_{\hat{a}}^{\hat{b}} E_{\hat{b}} \hat{m}$. We refer the reader to references $[12,14]$ for more details of the formalism and notation.

Using the solution of [15] and adopting the notation of [11] we consider a multi-threebrane solution which lies in the $\left(x^{0}, x^{1}, x^{2}, x^{3}\right)$ plane. We take only two transverse fields $X^{6}$ and $X^{10}$ to be active and assume that $X^{10}$ is a compact dimension of radius $R$. The other three scalars are constant and the three form vanishes. First let us consider the dependence of the fields on the M-fivebrane coordinates transverse to the threebranes, $x^{4}$ and $x^{5}$. This solution preserves half of the six-dimensional supersymmetries if [15]

$$
\partial_{4} X^{6}=\partial_{5} X^{10}, \quad \partial_{5} X^{6}=-\partial_{4} X^{10}
$$

This leads to an $N=2$ vector mulitplet on the four-dimensional worldvolume of the threebranes [15]. Adopting the complex notation $s=\left(X^{6}+i X^{10}\right) / R$ and $z=\Lambda^{2}\left(x^{4}+i x^{5}\right)$, where $\Lambda$ is a mass scale, we recognise (2.2) as the CauchyRiemann equation. Thus $s$ is a complex function of $z$ only. Furthermore any 
choice of this function will solve the field equations [15]. The threebranes can be then thought of as an M-fivebrane in an eight-dimensional space with coordinates $x^{0}, \ldots, x^{5}, X^{6}, X^{10}$, in which $x^{0}, \ldots, x^{3}$ are flat $\mathbf{R}^{4}$ and $x^{4}, x^{5}, X^{6}, X^{10}$ form a nontrivial four-dimensional space $Q$. The M-fivebrane field equations in the presence of these threebranes then imply that the M-fivebrane is wrapped on a Riemann surface $\Sigma$, which is embedded in the four-dimensional space $Q$. The volume of this Riemann surface is set by the scale $R^{2}$. Given the geometrical construction of the Riemann surface presented here, it is perhaps more natural to assign $z$ the dimensions of length. However we have assigned $z$ the dimensions of mass in order to make contact with the literature on the Seiberg-Witten solution.

Following [11] we define $t=e^{-s}$ and consider a threebrane configuration defined by

$$
F(t, z)=0
$$

where $F$ is a complex polynomial. In order to make contact with [11] let us consider the IIA picture in ten dimensions obtained by taking the small $R$ limit. In this case there are D-fourbranes in the $\left(x^{0}, x^{1}, x^{2}, x^{3}, X^{6}\right)$ plane, located at the roots of $F(\cdot, z)=0$ and also NS-fivebranes in the $\left(x^{0}, x^{1}, x^{2}, x^{3}, x^{4}, x^{5}\right)$ plane, located at the roots of $F(t, \cdot)=0$ [11]. The threebrane is the intersection of the D-fourbranes with the NS-fivebranes. The restriction to a polynomial $F$ then ensures that there are only a finite number of branes.

A simple example of such a configuration is a collection of $N$ threebranes at the positions $d_{i}, i=1, \ldots, N$, with charges $q_{i}[15]$

$$
s=s_{0}-\sum_{i} q_{i} \ln \left(z-d_{i}\right)
$$

where $s_{0}$ is an aribitrary constant which we set to zero. The corresponding surface is

$$
F(t, z) \equiv t^{2}-\prod_{i}\left(z-d_{i}\right)^{2 q_{i}}=0,
$$

where $t$ has been suitably rescaled by $\Lambda$. For integer values of $q_{i}$ this is a singular 
Riemann surface, with degenerate roots, while if $q_{i}=\frac{1}{2}$ it is non-singular. In fact we will use a more general threebrane configuration below.

The scalar fields in the resulting four-dimensional theory in $\mathbf{R}^{4}$ are the positions of the threebranes $d_{i}$. We therefore allow $s$ to be function of $x^{\mu}, \mu=0,1,2,3$ by letting the locations of the threebranes become $x^{\mu}$ dependent. As seen from the M-fivebrane this corresponds to letting the moduli of the Riemann Surface depend on $x^{\mu}$. We now wish to evaluate the dynamics for this configuration. For simplicity we will just consider the scalar fields with the three form field of the M-fivebrane vanishing, or equivalently with the vector fields in the four-dimensional effective theory set to zero.

For such a configuration the equation of motion is $g^{\hat{m} \hat{n}} \nabla_{\hat{m}} \nabla_{\hat{n}} s=0$ and can be derived from the usual brane action

$$
I_{5}=\int d^{6} x \sqrt{-\operatorname{det} g_{\hat{m} \hat{n}}}
$$

where, for a threebrane configuration,

$$
g_{\hat{m} \hat{n}}=\eta_{\hat{m} \hat{n}}+\frac{1}{2}\left(\partial_{\hat{m}} s \partial_{\hat{n}} \bar{s}+\partial_{\hat{m}} \bar{s} \partial_{\hat{n}} s\right)
$$

One can then evaluate the determinant in (2.6) to find

$$
-\operatorname{det} g_{\hat{m} \hat{n}}=\left(1+\frac{1}{2} \partial_{\hat{m}} s \partial^{\hat{m}} \bar{s}\right)^{2}-\frac{1}{4}\left|\partial_{\hat{m}} s \partial^{\hat{m}} s\right|^{2}
$$

In the above expressions and below we have suppressed factors of $R$ which appear with $s$. To obtain the low energy effective action for the threebrane we will only keep terms of second order in the derivatives $\partial_{\mu}$. Using the equation of motion of of $s$ one readily finds

$$
I_{5}=\frac{1}{2} \int d^{6} x \partial_{\mu} s \partial^{\mu} \bar{s}
$$

Let us now denote the moduli of the Riemann surface by $u_{i}=u_{i}(x)$. The above 
action becomes

$$
I_{5}=\frac{1}{4 i} \int d^{4} x \partial_{\mu} u_{i} \partial^{\mu} \bar{u}_{j} \int_{\Sigma} \omega^{i} \wedge \bar{\omega}^{j}
$$

where $\omega^{i}=-\partial s / \partial u_{i} d z$. To evaluate this action further we must adopt a particular form for the threebrane configuration and hence the Riemann surface $\Sigma$.

We wish to consider threebrane configurations which are everywhere smooth, so that the associated Riemann surface is also smooth, but which asymptotically take the form (2.4). To this end for the rest of this paper we shall restrict our attention to functions $F$ of the form

$$
F(t, z)=t^{2}-2 B(z) t+\Lambda^{2 N}=0
$$

where $B(z)$ is a polynomial of degree $N \geq 2$ and $t$ has again been suitably rescaled by $\Lambda$. However, it is also possible to consider more complicated threebrane configurations. The choice (2.11) leads to the scalar function

$$
s=s_{0}-\ln (B+\sqrt{Q})
$$

where we have introduced the polynomial $Q=B^{2}(z)-\Lambda^{2 N}$ and $s_{0}$ is a constant determined by $N$ and $\Lambda$. A sufficiently general parameterisation of $B(z)$ in terms of the $u_{i}$ 's is

$$
B(z)=z^{N}+u_{N-1} z^{N-2}+u_{N-2} z^{N-3}+\ldots+u_{1}
$$

Note that only the $z^{N-1}$ term is missing from (2.13). This is because, as noted in [11], such a term would cause the integral over the Riemann surface in (2.9) to diverge.

Lets us pause for a moment to consider the physical interpretation of this choice of $F$ in terms of the threebranes. If $\Lambda=0$ then this solution is described by $(2.4)$ 
with all the $q_{i}=1$ and the positions $d_{i}$ of the threebranes are the $N$ roots of $B$. From this point of view a $z^{N-1}$ term in (2.13) corresponds to the centre of mass coordinate for the threebranes (its coefficient $u_{N}$ is the sum of the roots). Low energy motion in this moduli would produce an infinite contribution to the action (2.9) and so has been frozen out. For a non-zero value of $\Lambda$ the Riemann surface is generically non-singular, but the picture in terms of the simple threebranes of (2.4) is slightly obscured. For $|z|>>\Lambda$ we again see $N$ distinct threebranes located at $d_{i}$ but for finite $z$ this is not the case. In fact one can see from (2.11) that there are no points $t=0$, corresponding to the singular core of a threebrane. In terms of the ten-dimensional type IIA picture (2.11) corresponds to two NS-fivebranes with $N$ D-fourbranes suspended between them [11].

We now evaluate the effective action (2.9). As explained in [11], the Riemann surface can be defined by

$$
\widetilde{t}^{2}=Q(z)=B^{2}(z)-\Lambda^{2 N}
$$

where $\tilde{t}=t-B$. It is a straightforward calculation to see that

$$
\omega^{i}=-\frac{\partial s}{\partial u_{i}} d z=\frac{z^{i-1}}{\sqrt{Q}}=\lambda^{i}
$$

where $\lambda^{i}$ is the $i$ th holomorphic form of the Riemann surface. Using the Riemann bi-linear relation we may express the effective action as

$$
I_{5}=\frac{1}{4 i} \int d^{4} x \partial_{\mu} u_{i} \partial^{\mu} \bar{u}_{j} \sum_{k=1}^{N-1}\left(\int_{A_{k}} \lambda^{i} \int_{B^{k}} \bar{\lambda}^{j}-\int_{A_{k}} \bar{\lambda}^{j} \int_{B^{k}} \lambda^{i}\right)
$$

where $A_{k}$ and $B^{k}$ are a basis for the $a$ and $b$ cycles on the Riemann surface.

Next we note that $\partial_{\mu} u_{i} \lambda^{i}=\partial_{\mu} \lambda_{S W}$, where we have introduced the Seiberg- 
Witten differential $\lambda_{S W}$ which satisfies [1]

$$
\frac{\partial \lambda_{S W}}{\partial u_{i}}=\lambda^{i}
$$

Therefore if we define the new scalar modes

$$
a_{i}=\int_{A_{i}} \lambda_{S W}, \quad a_{D}^{i}=\int_{B^{i}} \lambda_{S W},
$$

we obtain the effective action

$$
\begin{aligned}
I_{5} & =\frac{1}{4 i} \int d^{4} x\left(\partial_{\mu} a_{k} \partial^{\mu} \bar{a}_{D}^{k}-\partial_{\mu} \bar{a}_{k} \partial^{\mu} a_{D}^{k}\right), \\
& =-\frac{1}{2} \operatorname{Im}\left(\int d^{4} x \partial_{\mu} \bar{a}_{i} \partial^{\mu} a_{j} \tau^{i j}\right) .
\end{aligned}
$$

In (2.19) we have introduced $\tau^{i j}=\partial a_{D}^{i} / \partial a_{j}$, which on the general grounds of $N=2$ supersymmetry may be obtained from the holomorphic prepotential $\mathcal{F}$ as $\tau^{i j}=\partial^{2} \mathcal{F} / \partial a_{i} \partial a_{j}[1]$. It was shown in [11] that the brane configuration we are considering produces the correct Riemann surface and hence the correct SeibergWitten differential for $N=2 S U(N)$ Yang-Mills [9]. Thus we have arrived at the scalar part of the full Seiberg-Witten effective action for $N=2 S U(N)$ YangMills theory. Furthermore as a consequence of $N=2$ supersymmetry $\mathcal{F}$ uniquely determines the entire low energy effective theory, including the terms that we would have obtained by considering the fermionic and and vector zero modes. Thus the effective action for all of the threebrane zero modes is the complete Seiberg-Witten effective action. We leave the details of this calculation to be performed elsewhere.

Finally we would like to point out that in obtaining the Seiberg-Witten effective action we have discarded various higher derivative terms in (2.6). If we include these the full action for the scalars is

$$
\begin{aligned}
I_{5} & =\int d^{4} x \int_{\Sigma} d^{2} z \sqrt{\left(1+\Lambda^{4} R^{2}\left|\partial_{z} s\right|^{2}+\frac{1}{2} R^{2} \partial_{\mu} s \partial^{\mu} \bar{s}\right)^{2}-\frac{1}{4} R^{4}\left|\partial_{\mu} s \partial^{\mu} s\right|^{2}} \\
& =I_{S W}-\frac{R^{4}}{8} \int d^{4} x \int_{\Sigma} d^{2} z \frac{\left|\partial_{\mu} s \partial^{\mu} s\right|^{2}}{1+R^{2} \Lambda^{4}\left|\partial_{z} s\right|^{2}}+\mathcal{O}\left(\left|\partial_{\mu} s\right|^{6}\right)
\end{aligned}
$$


where $I_{S W}$ is the Seiberg-Witten effective action (2.19) and we have included all the factors of $R$. By writing $\partial_{\mu} s=\lambda^{i} \partial_{\mu} u_{i}$ and continuing the expansion in (2.20) one obtains an infinite series of higher derivative terms involving powers of $\lambda^{i}$. It is natural to suppose that these terms correspond to some of the higher derivative corrections to the Seiberg-Witten effective action. Note that the terms in (2.20) have a particular form; where each $u_{i}$ has one and only one derivative acting on it. One may hope that these are all of the higher derivative terms of this type.

\section{Discussion}

In this paper we have considered the $\mathrm{M}$ theory fivebrane with threebranes moving within it. We evaluated the classical M-fivebrane equations of motion for this field configuration when the zero modes of the threebrane solutions are taken to depend on $x^{\mu}$, the four-dimensional coordinates of threebrane worldvolume. The resulting four-dimensional theory corresponds to a spontaneously broken $N=2$ gauge theory and the corresponding low energy effective action is that given by Seiberg and Witten. Hence we have derived the complete quantum nonperturbative Seiberg-Witten effective action from the classical dynamics of the M theory fivebrane.

Given our conventional understanding of the relationship between classical and quantum theories it is surprising to see such a detailed and complete connection. Although M theory has only one scale $M_{\text {Planck, }}$, the solution for the threebranes introduces two more scales $R$ and $\Lambda$, which occur as integration constants. Reintroducing $\hbar$ we would find that it occurs in combination with these integration constants. Since these constants and so $\hbar$ do not play the rôle of a perturbative parameter in the derivation we have given, from the $M$ theory perspective it is perhaps not so surprising to have found the full non-perturbative Seiberg-Witten effective action.

We note that we have only obtained the classical effective action for the threebrane collective coordinates, which one must then start to quantise. This corre- 
sponds to the fact that the Seiberg-Witten effective action is obtained by integrating out the massive modes in the path integral. However in the full quantum theory one is still left with the integral over the massless $U(1)^{N-1}$ modes. Along these lines one may also wonder how the non-Abelian structure of $S U(N)$ Yang-Mills can be seen from the M-fivebrane perspective.

Finally, although we have used the constraints of $N=2$ supersymmetry to deduce the full Seiberg-Witten effective action from only its scalar part, this was not crucial. We could also have explicitly derived these terms by considering the collective coordinates of the three form. Thus one may wonder if the proceedure considered here can be applied to situations with less supersymmetry, perhaps leading to effective actions for some $N=1$ supersymmetric theories.

P.C.W. would like to thank D.I. Olive for discussions.

\section{REFERENCES}

1. N. Seiberg and E. Witten, Nucl. Phys. B426 (1994) 19, hep-th/9407087; Nucl. Phys. B431 (1994) 484, hep-th/9408099

2. P. Howe, K. Stelle and P. West, Phys. Lett. 124B (1983) 55. For a review of perturbative results in rigid supersymmetric theories see P. West, Supersymmety and Finiteness, in Proceedings of the 1983 Shelter Island II Conference on Quntum Field Theory and Fundamental Problems of Physics, edited by R. Jackiw, N. Kuri, S. Weinberg and E. Witten (M.I.T. Press)

3. N. Seiberg, Phys. Lett. 318B (1993) 469

4. C. Montonen and D. Olive, Phys. Lett. 72B (1977) 117

5. N. Dorey, V. V. Khoze and M. P. Mattis, Phys. lett. B388 (1996) 324, hep-th/9607066; Phys. Rev. D54 (1996) 2921; Phys. Rev. D54 (1996) 7832, hep-th/9607202; K. Ito and N. Sasakura, Phys. Lett. B382 (1996) 95, hepth/9602073; Nucl. Phys. B484 (1997) 141, hep-th/9608054; A. Yung, Nucl. Phys. B485 (1997) 38, hep-th/9605096; H. Aoyama, T. Harano, M. Sato 
and S. Wada, Phys. lett. B388 (1996) 331, hep-th/9607076; T. Harano and M. Sato, Nucl.Phys. B484 (1997) 167, hep-th/9608060

6. M. Mantone, Phys. Lett. 357B (1995) 342, hep-th/9506102

7. P. Howe and P. West, Nucl. Phys. B486 (1997) 425, hep-th/9607239

8. M. Magro, L. O’Raifeartaigh and I. Sachs, Seiberg-Witten Effective Lagrangian from Superconformal Ward Identities, hep-th/9704027

9. P. Argyres and A. Faraggi, Phys. Rev. Lett. 74 (1995) 3931, hep-th/9411057; S. Klemm, W. Lerche, S. Theisen and S. Yankielowicz, Phys. Lett. 344B (1995) 169, hep-th/9411048

10. S. Kachru and C. Vafa, Nucl. Phys. B450 (1995) 69, hep-th/9505105; S. Kachru, A. Klemm, W. Lerche, P. Mayr and C. Vafa, Nucl. Phys. B359 (1995) 537, hep-th/9508155; A. Klemm, W. Lerche, P. Mayr, C. Vafa and N. Warner, Nucl. Phys. B477 (1996) 746, hep-th/9604034

11. E. Witten, Solutions of Four Dimensional Field Theories via M Theory, hep-th/97003166

12. P.S. Howe, E. Sezgin and P.C. West, Phys. Lett. B399 (1997) 49, hepth/9702008

13. M. Perry and J.H. Schwarz, Nucl. Phys. B489 (1997) 47, hep-th/9611065; M. Aganagic, J. Park, C. Popescu, and J. H. Schwarz, Worldvolume action of the M-theory fivebrane, hep-th/9701166; I. Bandos, K Lechner, A. Nurmagambetov, P. Pasti and D. Sorokin, and M. Tonin, Covariant action for the super fivebrane of M-theory, hep-th/9701149

14. P.S. Howe, N.D. Lambert and P.C. West, The Self-Dual String Soliton, hep-th/9709014

15. P.S. Howe, N.D. Lambert and P.C. West, The Threebrane Soliton of the M-Fivebrane, hep-th/9710033 